\title{
ITERATED COLLOCATION METHODS FOR VOLTERRA INTEGRAL EQUATIONS WITH DELAY ARGUMENTS
}

\author{
HERMANN BRUNNER
}

\begin{abstract}
In this paper we give a complete analysis of the global convergence and local superconvergence properties of piecewise polynomial collocation for Volterra integral equations with constant delay. This analysis includes continuous collocation-based Volterra-Runge-Kutta methods as well as iterated collocation methods and their discretizations.
\end{abstract}

\section{INTRODUCTION}

In this paper we analyze the numerical discretization of Volterra integral equations with (constant) delay $\tau>0$,

$$
\begin{aligned}
y(t)= & g(t)+\int_{0}^{t} k_{1}(t, s, y(s)) d s \\
& +\int_{0}^{t-\tau} k_{2}(t, s, y(s)) d s, \quad t \in I:=[0, T],
\end{aligned}
$$

with

$$
y(t)=\phi(t), \quad t \in[-\tau, 0),
$$

by collocation methods in certain (nonsmooth) piecewise polynomial spaces. Equation (1.1) encompasses an important particular delay equation frequently encountered in physical and biological modelling processes [13], namely

$$
y(t)=g(t)+\int_{t-\tau}^{t} k(t, s, y(s)) d s, \quad t \in I
$$

it corresponds to setting $k_{2}=-k_{1}(=k)$ in (1.1).

It will be assumed that the given functions, $\phi:[-\tau, 0] \longrightarrow \mathbf{R}, g: I \longrightarrow \mathbf{R}$, $k_{1}: S \times \mathbf{R} \longrightarrow \mathbf{R}(S:=\{(t, s): 0 \leq s \leq t \leq T\})$, and $k_{2}: S_{\tau} \times \mathbf{R} \longrightarrow \mathbf{R}$ $\left(S_{\tau}:=I \times[-\tau, T-\tau]\right)$ are (at least) continuous on their domains; additional conditions will be imposed later when needed. In order to exclude "classical" Volterra integral equations (which were considered in $[6,8]$ ) we also assume

Received by the editor July 21, 1992.

1991 Mathematics Subject Classification. Primary 65R20, 45L10.

This work was supported by the Natural Sciences and Engineering Research Council of Canada (Operating Grant A9406). 
that $k_{2}(t, s, y)$ does not vanish identically. Existence and uniqueness results for (1.1) and for related Volterra integral equations with finite delay (e.g.,

$$
y(t)=g(t)+\int_{0}^{t} k(t, s, y(s), y(\alpha(s))) d s, \quad t \in I,
$$

with $\alpha(s)<s)$ can be found, for example, in [3, 7, 12, 13, 14].

In recent years, various aspects of numerical methods for (1.1), (1.3), or (1.4) have been studied. Results dealing with convergence properties are given in [17] (Euler's method, trapezoidal and midpoint method for (1.4) with pure delay and $\alpha(s)=s-\tau$ ); [14] (Hermite-type collocation for (1.4)); [12] (direct quadrature methods for (1.4) with state-dependent delay: $\alpha=\alpha(y(s)))$; [1] (extension of ODE Runge-Kutta methods to (1.3)); and [19] (general RungeKutta methods and their natural extensions for (1.1)). The stability analyses for Runge-Kutta-type methods [2, 19] and $(\rho, \sigma)$-reducible quadrature methods [11] are restricted to (1.1) with $k_{1}(t, s, y)=a, k_{2}(t, s, y)=b(a, b$ constants), with $|b| \leq-\operatorname{Re}(a)$. Note that in this case (1.1) can be reduced to a delay differential equation with constant coefficients,

$$
y^{\prime}(t)=a y(t)+b y(t-\tau), \quad t \in I,
$$

(assuming that $g(t)=y_{0}=$ const).

In [19] the stability of collocation methods is briefly discussed. However, an analysis of the global convergence and the local superconvergence properties of collocation methods for (1.1) is essentially still lacking. (However, in [14] Hermite-type collocation in the space $S_{2 m-1}^{(m-1)}\left(\Pi_{N}\right)$ (piecewise polynomials of degree $2 m-1$ which are in $C^{m-1}(I)$; see $\S 2$ for details on notation) is studied and shown to yield global $O\left(h^{2 m}\right)$-convergence.) It is the aim of this paper to show that $O\left(h^{2 m}\right)$-convergence at the mesh points $\Pi_{N}$ can be attained by using the iterated collocation solution corresponding to collocation in $S_{m-1}^{(-1)}\left(\Pi_{N}\right)$ (piecewise polynomials of degree $m-1$ possessing jump discontinuities on $\Pi_{N}$ ); on the interval $I$ itself, the order of convergence will turn out to be $p=m$.

\section{COLLOCATION AND ITERATED COLLOCATION}

Let $t_{n}:=n h\left(n=0, \ldots, N-1 ; t_{N}=T\right)$ define a uniform partition for $I=[0, T]$, and set $\Pi_{N}:=\left\{t_{0}, \ldots, t_{N}\right\}, I_{0}:=\left[t_{0}, t_{1}\right], \quad I_{n}:=\left(t_{n}, t_{n+1}\right](1 \leq$ $n \leq N-1)$. The mesh $\Pi_{N}$ is assumed to be constrained, i.e.,

$$
h=\frac{\tau}{r} \text { for some } r \in \mathbf{N} \text {. }
$$

For given integers $d \geq-1$ and $m \geq 1$ the piecewise polynomial space $S_{m+d}^{(d)}\left(\Pi_{N}\right)$ is defined by

$$
\begin{aligned}
S_{m+d}^{(d)}\left(\Pi_{N}\right):=\left\{u: I \longrightarrow \mathbf{R} ;\left.u\right|_{I_{n}}=: u_{n} \in \pi_{m+d} ;\right. \\
\left.u_{n-1}^{(\nu)}\left(t_{n}\right)=u_{n}^{(\nu)}\left(t_{n}\right), \nu=0, \ldots, d\right\},
\end{aligned}
$$


where $\pi_{m+d}$ denotes the set of (real) polynomials of degree not exceeding $m+d$. The dimension of this vector space is obviously given by

$$
\operatorname{dim} S_{m+d}^{(d)}\left(\Pi_{N}\right)=N m+d+1
$$

This shows, in the context of collocation, that the natural choice of $d$ in (2.2) will be governed by the nature of the functional equation to be solved: if the equation under consideration is a differential or integro-differential equation of order $\kappa$, then $d=\kappa-1$; when solving integral equations like (1.1) we choose $d=-1$.

For given real numbers $\left\{c_{j}\right\}$ with $0 \leq c_{1}<\cdots<c_{m} \leq 1$, define the set $X_{N}:=\left\{t_{n, j}\right\}$ of collocation points by

$$
t_{n, j}:=t_{n}+c_{j} h \quad(j=1, \ldots, m ; n=0, \ldots, N-1) .
$$

The collocation solution $u \in S_{m-1}^{(-1)}\left(\Pi_{N}\right)$ to (1.1) is then given by the equation

$$
u(t)=g(t)+\int_{0}^{t} k_{1}(t, s, u(s)) d s+\int_{0}^{t-\tau} k_{2}(t, s, u(s)) d s, \quad t \in X_{N},
$$

with

$$
u(t)=\phi(t) \text { on }[-\tau, 0) .
$$

If $t=t_{n, j}$ is such that $t_{n, j}-\tau \quad\left(=t_{n-r, j}\right)<0$ (recall that, by (2.1), $\tau=r h=$ $\left.t_{r}\right)$, then (2.4) becomes

$$
u(t)=g(t)+\int_{0}^{t} k_{1}(t, s, u(s)) d s-\Phi(t), \quad t=t_{n, j}
$$

$(j=1, \ldots, m ; n=0, \ldots, r-1)$, where

$$
\Phi(t):=\int_{t-\tau}^{0} k_{2}(t, s, \phi(s)) d s .
$$

In contrast to classical Volterra integral equations corresponding to $k_{2}=0$ (where no initial condition is present), or delay differential equations (where collocation requires the evaluation of the given initial function at certain points), the occurrence of the term $\Phi(t)$ in the collocation equation (2.6) reveals that, for $t=t_{n, j}<\tau$, we have to evaluate (or approximate) a functional containing the given initial function $\phi(t)$. As will be shown in $\S 3$, this represents a further potential source of error.

The iterated collocation solution, $u_{i t}$, based on the collocation solution $u$ defined by (2.4), (2.5) is given by

$$
u_{i t}(t):=g(t)+\int_{0}^{t} k_{1}(t, s, u(s)) d s+\int_{0}^{t-\tau} k_{2}(t, s, u(s)) d s, \quad t \in I .
$$

It has the property that

$$
u_{i t}(t)=u(t) \quad \text { whenever } t \in X_{N} .
$$


In order to put (2.4) and (2.8) into a form amenable to numerical computation, let $t \in I_{n}$, and define

$$
\begin{aligned}
F_{n}(t) & :=\int_{0}^{t_{n}} k_{1}(t, s, u(s)) d s \\
& =h \sum_{i=0}^{n-1} \int_{0}^{1} k_{1}\left(t, t_{i}+v h, u\left(t_{i}+v h\right)\right) d v .
\end{aligned}
$$

Moreover, set

$$
D(t):=\int_{0}^{t-\tau} k_{2}(t, s, u(s)) d s
$$

(with $D(t)=-\Phi(t)$ if $t<\tau)$. Since $u \in \pi_{m-1}$ on $I_{n}$, we may write

$$
u\left(t_{n}+v h\right)=\sum_{k=1}^{m} L_{k}(v) U_{n, k}, \quad t_{n}+v h \in I_{n},
$$

where $U_{n, k}:=u\left(t_{n, k}\right)$ and

$$
L_{k}(v):=\prod_{l \neq k}^{m} \frac{v-c_{l}}{c_{k}-c_{l}} .
$$

Thus, (2.4) assumes the form

$$
\begin{aligned}
U_{n, j}= & g\left(t_{n, j}\right)+F_{n}\left(t_{n, j}\right)+D\left(t_{n, j}\right) \\
& +h \int_{0}^{c_{j}} k_{1}\left(t_{n, j}, t_{n}+v h, \sum_{k=1}^{m} L_{k}(v) U_{n, k}\right) d v,
\end{aligned}
$$

$j=1, \ldots, m$. For each $n=0, \ldots, m,(2.12)$ represents a system of $m$ nonlinear algebraic equations for $U_{n}:=\left(U_{n, 1}, \ldots, U_{n, m}\right)^{T} \in \mathbf{R}^{m}$. For $t=$ $t_{n}+z h \in I_{n}$, the iterated collocation equation (2.8) becomes

$$
\begin{aligned}
u_{i t}(t)= & g(t)+F_{n}(t)+D(t) \\
& +h \int_{0}^{z} k_{1}\left(t, t_{n}+v h, \sum_{k=1}^{m} L_{k}(v) U_{n, k}\right) d v .
\end{aligned}
$$

Consider now (2.12): in general, the integrals on the right-hand side (including those in $F_{n}\left(t_{n, j}\right)$ and $\left.D\left(t_{n, j}\right)\right)$ cannot be evaluated analytically but have to be approximated by suitable quadrature formulas. We choose interpolatory $m$ point quadrature formulas whose abscissas are given by the collocation points. Specifically,

$$
\int_{0}^{c_{j}} k_{1}\left(t_{n, j}, t_{n}+v h, u\left(t_{n}+v h\right)\right) d v
$$

will be replaced by

$$
\sum_{l=0}^{m} w_{j, l} k_{1}\left(t_{n, j}, t_{n}+c_{j} c_{l} h, u\left(t_{n}+c_{j} c_{l} h\right)\right)
$$


with $w_{j, l}:=c_{j} w_{l}, w_{l}=\int_{0}^{1} L_{l}(v) d v$, and $u\left(t_{n}+c_{j} c_{l} h\right)$ given by (2.11), with $v=c_{j} c_{l}$.

The resulting fully discretized collocation equation corresponding to (2.12) is thus given by

$$
\begin{aligned}
\hat{U}_{n, j}= & g\left(t_{n, j}\right)+\hat{F}_{n}\left(t_{n, j}\right)+\hat{D}\left(t_{n, j}\right) \\
& +h c_{j} \sum_{l=1}^{m} w_{l} k_{1}\left(t_{n, j}, t_{n}+c_{j} c_{l} h, \sum_{k=1}^{m} L_{k}\left(c_{j} c_{l}\right) \hat{U}_{n, k}\right),
\end{aligned}
$$

$(j=1, \ldots, m)$, with

$$
\hat{F}_{n}(t):=h \sum_{i=0}^{n-1} \sum_{l=1}^{m} k_{1}\left(t, t_{i, l}, \hat{U}_{i, l}\right)
$$

and

$$
\begin{aligned}
\hat{D}\left(t_{n, j}\right):= & h \sum_{i=0}^{n-r-1} \sum_{l=1}^{m} k_{2}\left(t_{n, j}, t_{i, l}, \hat{U}_{i, l}\right) \\
& +h c_{j} \sum_{l=1}^{m} w_{l} k_{2}\left(t_{n, j}, t_{n-r}+c_{j} c_{l} h, \sum_{k=1}^{m} L_{k}\left(c_{j} c_{l}\right) \hat{U}_{n-r, k}\right),
\end{aligned}
$$

provided that $n-r \geq 0$. If $n-r<0$, then $\hat{D}\left(t_{n, j}\right)$ is given either by the exact value of $-\Phi\left(t_{n, j}\right)$ (recall (2.7)),

$$
\begin{aligned}
\hat{D}\left(t_{n, j}\right)= & D\left(t_{n, j}\right)=-h \int_{c_{j}}^{1} k_{2}\left(t_{n, j}, t_{n-r}+v h, \phi\left(t_{n-r}+v h\right)\right) d v \\
& -h \sum_{i=n-r+1}^{-1} \int_{0}^{1} k_{2}\left(t_{n, j}, t_{i}+v h, \phi\left(t_{i}+v h\right)\right) d v
\end{aligned}
$$

or by a suitable quadrature approximation to $-\Phi\left(t_{n, j}\right)$, e.g., by

$$
\begin{aligned}
\hat{D}\left(t_{n, j}\right)= & -h \sum_{l=1}^{m} \tilde{w}_{j, l} k_{2}\left(t_{n, j}, t_{n-r}+\xi_{j, l} h, \phi\left(t_{n-r}+\xi_{j, l} h\right)\right) \\
& -h \sum_{i=n-r+1}^{-1} \sum_{l=1}^{m} w_{l} k_{2}\left(t_{n, j}, t_{i, l}, \phi\left(t_{i, l}\right)\right),
\end{aligned}
$$

where

$$
\xi_{j, l}:=c_{j}+\left(1-c_{j}\right) c_{l}, \quad \tilde{w}_{j, l}:=\left(1-c_{j}\right) w_{l} \quad(j, l=1, \ldots, m) .
$$

The fully discretized collocation scheme generates a collocation solution $\hat{u} \in$ $S_{m-1}^{-1}\left(\Pi_{N}\right)$, with $\varepsilon:=u-\hat{u} \neq 0$ in general.

Equations (2.14)-(2.16), together with (2.11) $(v=1)$,

$$
\hat{y}_{n+1}:=\hat{u}\left(t_{n+1}\right)=\sum_{k=1}^{m} L_{k}(1) \hat{U}_{n, k}
$$


represent an implicit $m$-stage Volterra-Runge-Kutta (DVRK) method for the delay integral equation (1.1). This class of collocation-based Runge-Kutta methods forms an important subset of the general DVRK methods discussed in [19].

The fully discretized version of the iterated collocation equation (2.13) becomes, for $t=t_{n+1}$,

$$
\begin{aligned}
\hat{u}_{i t}\left(t_{n+1}\right)= & g\left(t_{n+1}\right)+\hat{F}_{n}\left(t_{n+1}\right)+\hat{D}\left(t_{n+1}\right) \\
& +h \sum_{l=1}^{m} w_{l} k_{1}\left(t_{n+1}, t_{n, l}, \hat{U}_{n, l}\right) ;
\end{aligned}
$$

for arbitrary values of $t=t_{n}+z h \in I_{n}$, it is defined by an expresssion similar to the right-hand side of $(2.14)$, with $z$ replacing $c_{j}$.

\section{Global convergence}

Let $u \in S_{m-1}^{(-1)}\left(\Pi_{N}\right)$ denote the (exact) collocation solution to (1.1) defined by (2.4)-(2.6). For ease of exposition we will focus on the linear version of (1.1),

$$
y(t)=g(t)+\int_{0}^{t} K_{1}(t, s) y(s) d s+\int_{0}^{t-\tau} K_{2}(t, s) y(s) d s, \quad t \in I,
$$

where $K_{1} \in C(S), \quad K_{2} \in C\left(S_{\tau}\right)$. A comment on the extension of the convergence results to the nonlinear equation (1.1) can be found at the end of the section.

Theorem 3.1. Assume that the given functions in (3.1) and (1.2) satisfy $g \in$ $C^{m}(I), K_{1} \in C^{m}(S), K_{2} \in C^{m}\left(S_{\tau}\right), \phi \in C^{m}[-\tau, 0]$, and that, for $t \in[0, \tau]$, the integral (2.7),

$$
\Phi(t):=\int_{t-\tau}^{0} K_{2}(t, s) \phi(s) d s
$$

is known exactly. Then for all sufficiently small $h=\tau / r(r \in \mathbf{N})$ the constrainedmesh collocation solution $u \in S_{m-1}^{(-1)}$ to (3.1) satisfies

$$
\|y-u\|_{\infty} \leq C h^{m}
$$

for some finite $C$ not depending on $h$. This estimate holds for all collocation parameters $\left\{c_{j}\right\}$ with $0 \leq c_{1}<\cdots<c_{m} \leq 1$.

The iterated collocation solution corresponding to u exhibits the same global order of convergence,

$$
\left\|y-u_{i t}\right\|_{\infty} \leq C h^{m} .
$$

Proof. Assume for simplicity, and without any loss of generality, that $T=M \tau$ for some $M \in \mathbf{N}$. In each interval $J_{\mu}:=(\mu \tau,(\mu+1) \tau)$, the exact solution $y$ of (3.1) is $m$ times continuously differentiable. This follows from the smoothness hypotheses we have imposed on $\phi, g, K_{1}$, and $K_{2}$, and from the expressions for $y^{(\nu)}(t)$ obtained by successively differentiating (3.1) with respect to $t$. From this it is readily seen that both the left and right limits of $y^{(\nu)}(t)(\nu=0, \ldots, m)$, as $t$ tends to $\mu \tau$, exist and are finite. Thus, let 
$t=t_{n}+v h \in I_{n}$ and set

$$
e\left(t_{n}+v h\right)=h^{m} \sum_{l=1}^{m} \beta_{n, l} v^{l-1}+h^{m} R_{n}(v)
$$

where

$$
y\left(t_{n}+v h\right)=\sum_{l=1}^{m} \gamma_{n, l} v^{l-1}+h^{m} R_{n}(v), \quad \gamma_{n, l}:=h^{l-1} y^{(l-1)}\left(t_{n}\right) /(l-1) !
$$

(with $R_{n}(v)$ denoting the remainder term in the above application of Taylor's formula),

$$
u\left(t_{n}+v h\right)=\sum_{l=1}^{m} \alpha_{n, l} v^{l-1}
$$

and

$$
h^{m} \beta_{n, l}:=\gamma_{n, l}-\alpha_{n, l} \quad(l=1, \ldots, m) .
$$

It follows from the above observation on the smoothness of the exact solution that at each breakpoint $t_{n}=\mu \tau(\mu=0, \ldots, M-1)$,

$$
y^{(l-1)}\left(t_{n}\right):=\lim _{t \downarrow t_{n}} y^{(l-1)}(t), \quad l=1, \ldots, m,
$$

exists and is finite.

The collocation error, $e:=y-u$, satisfies

$$
e\left(t_{n, j}\right)=\int_{0}^{t_{n, j}} K_{1}\left(t_{n, j}, s\right) e(s) d s+\int_{0}^{t_{n-r, j}} K_{2}\left(t_{n, j}, s\right) e(s) d s
$$

$j=1, \ldots, m(n=0, \ldots, N-1)$. If $t_{n}<\tau\left(=t_{r}\right)$, then $t_{n-r, j}=t_{n}+c_{j} h-$ $\tau \leq 0$. Since $u(t)=\phi(t)$ on $[-\tau, 0)$, equation (3.5) reduces to

$$
e\left(t_{n, j}\right)=\int_{0}^{t_{n, j}} K_{1}\left(t_{n, j}, s\right) e(s) d s
$$

which may be written as

$$
\begin{aligned}
e\left(t_{n, j}\right)= & h \int_{0}^{c_{j}} K_{1}\left(t_{n, j}, t_{n}+v h\right) e\left(t_{n}+v h\right) d v \\
& +h \sum_{i=0}^{n-1} \int_{0}^{1} K_{1}\left(t_{n, j}, t_{i}+v h\right) e\left(t_{i}+v h\right) d v .
\end{aligned}
$$

Using the expression (3.4), and setting $\beta_{n}:=\left(\beta_{n, 1}, \ldots, \beta_{n, m}\right)^{T} \in \mathbf{R}^{m}$, and $q_{n}:=\left(q_{n, 1}, \ldots, q_{n, m}\right)^{T} \in \mathbf{R}^{m}$, with

$$
\begin{aligned}
q_{n, j}:= & -R_{n}\left(c_{j}\right)+h \int_{0}^{c_{j}} K_{1}\left(t_{n, j}, t_{n}+v h\right) R_{n}(v) d v \\
& +h \sum_{i=0}^{n-1} \int_{0}^{1} K_{1}\left(t_{n, j}, t_{i}+v h\right) R_{i}(v) d v,
\end{aligned}
$$


we obtain a recurrence relation for the vectors $\beta_{n}$ of the form

$$
\left(V-h Q_{n, n}^{(1)}\right) \beta_{n}=h \sum_{i=0}^{n-1} Q_{n, i}^{(1)} \beta_{i}+q_{n} \quad(0 \leq n \leq r-1),
$$

where $V$ is the Vandermonde matrix $V=\left(c_{j}^{l-1}\right)$, and where the matrices $Q_{n, i}^{(1)}$ are given by

$$
Q_{n, n}^{(1)}:=\left(\int_{0}^{c_{j}} K_{1}\left(t_{n, j}, t_{n}+v h\right) v^{l-1} d v\right)
$$

and

$$
Q_{n, i}^{(1)}:=\left(\int_{0}^{1} K_{1}\left(t_{n, j}, t_{i}+v h\right) v^{l-1} d v\right) \quad(i<n) .
$$

Since by assumption, $K_{1} \in C(S)$, we have $\left\|h Q_{n, n}^{(1)}\right\|_{1}<1$ for all sufficiently small $h>0$, and so $V-h Q_{n, n}^{(1)}$ is nonsingular. It thus follows from (3.6) that

$$
\left\|\beta_{n}\right\|_{1} \leq h C_{0} \sum_{i=0}^{n-1}\left\|\beta_{i}\right\|_{1}+C_{1} \text {. }
$$

A well-known result on discrete Gronwall inequalities (see, e.g., [10, p. 41]) leads to

$$
\left\|\beta_{n}\right\|_{1} \leq C_{1} \cdot \exp \left(C_{0} \tau\right)=: B
$$

$(n=0, \ldots, r-1)$ uniformly as $h \rightarrow 0$ (where $r h=\tau)$. This implies, by (3.4), that

$$
\left|e\left(t_{n}+v h\right)\right| \leq h^{m}(B+R)=: C h^{m},
$$

for $v \in[0,1], n=0, \ldots, r-1$.

Now let $t_{n} \geq t_{r}(=\tau)$. Starting with (3.5), and using again the expression (3.4) for the collocation error on $I_{n}$, we find, in complete analogy to the above,

$$
\left(V-h Q_{n, n}^{(1)}\right) \beta_{n}=h \sum_{i=0}^{n-1} Q_{n, i}^{(1)} \beta_{i}+h \sum_{i=0}^{n-r} Q_{n, i}^{(2)} \beta_{i}+q_{n}
$$

$(n \geq r)$. Here, the matrices $Q_{n, i}^{(2)}$ are defined similarly to the matrices $Q_{n, i}^{(1)}$ introduced in (3.6), where now $0 \leq i \leq n-r$. Moreover, the expression for $q_{n}$ now contains additional terms involving the kernel $K_{2}$. We omit these obvious details.

For all sufficiently small $h>0$, equation (3.9) once more yields a discrete Gronwall inequality,

$$
\left\|\beta_{n}\right\|_{1} \leq h C_{0} \sum_{i=0}^{n-1}\left\|\beta_{i}\right\|_{1}+h D_{0} \sum_{i=0}^{n-r}\left\|\beta_{i}\right\|_{1}+C_{1},
$$

where now $r \leq n \leq N-1$. The estimate (3.8) and the result [10, p. 41] then imply that

$$
\left\|\beta_{n}\right\|_{1} \leq B \quad(r \leq n \leq N-1)
$$

uniformly as $h \rightarrow 0$ (with $N h=T$ ). Hence, by (3.4), the statement (3.2) of Theorem 3.1 follows. 
The error $e_{i t}:=y-u_{i t}$ associated with the iterated collocation solution introduced in (2.8) is given (for the linear case (3.1)) by

$$
e_{i t}(t)=\int_{0}^{t} K_{1}(t, s) e(s) d s+\int_{0}^{t-\tau} K_{2}(t, s) e(s) d s, \quad t \in I,
$$

with $e(t)=0$ on $[-\tau, 0]$. Hence, for $t \in[0, \tau]$, we obtain

$$
\left\|e_{i t}\right\|_{\infty} \leq\|e\|_{\infty} \cdot \max _{0 \leq t \leq T} \int_{0}^{t}\left|K_{1}(t, s)\right| d s \leq C_{0} h^{m},
$$

while for $t \in[\tau, T]$ we find

$$
\begin{aligned}
\left\|e_{i t}\right\|_{\infty} & \leq\|e\|_{\infty} \cdot\left(\max _{0 \leq t \leq T} \int_{0}^{t}\left|K_{1}(t, s)\right| d s+\max _{0 \leq t \leq T} \int_{0}^{t-\tau}\left|K_{2}(t, s)\right| d s\right) \\
& \leq C_{1} h^{m} .
\end{aligned}
$$

This completes the proof of Theorem 3.1.

We now turn to the case where the integral $\Phi(t)$ in (2.7) (see also (2.17)) cannot be found analytically but has to be approximated by suitable numerical quadrature.

Theorem 3.2. Let the assumptions of Theorem 3.1 hold, except that the integrals

$$
\Phi(t)=\int_{t-\tau}^{0} K_{2}(t, s) \phi(s) d s, \quad t=t_{n, j}(n=0, \ldots, r-1),
$$

are now approximated by quadrature formulas $\hat{\boldsymbol{\Phi}}(t)$, with corresponding quadrature errors $E_{0}(t):=\Phi(t)-\hat{\Phi}(t)$ such that

$$
\left|E_{0}(t)\right| \leq Q_{0} h^{q}, \quad t=t_{n, j}(0 \leq n<r),
$$

for some $q>0$. Then the collocation solution $u \in S_{m-1}^{(-1)}\left(\Pi_{N}\right)$ satisfies, for all sufficiently small $h>0$,

$$
\|e\|_{\infty} \leq C h^{p}, \quad \text { with } p:=\min \{m, q\} .
$$

The same convergence estimate holds for the iterated collocation solution $u_{i t}$, provided (3.10) is valid for all $t \in[-\tau, 0]$.

Proof. Recall the collocation equation (2.12), and assume that $0 \leq n<r$. If we subtract this equation from (3.1) (with $t=t_{n, j}$ ), we find

$$
e\left(t_{n, j}\right)=\int_{0}^{t_{n, j}} K_{1}\left(t_{n, j}, s\right) e(s) d s-\left(\boldsymbol{\Phi}\left(t_{n, j}\right)-\hat{\boldsymbol{\Phi}}\left(t_{n, j}\right)\right),
$$

where $\Phi(t)-\hat{\Phi}(t)=E_{0}(t)$. Instead of (3.4), write

$$
e\left(t_{n}+v h\right)=h^{p} \sum_{l=1}^{m} \beta_{n, l} v^{l-1}+h^{m} R_{n}(v),
$$

with suitable $p>0$ to be determined, and with

$$
h^{p} \beta_{n, l}:=\gamma_{n, l}-\alpha_{n, l} \quad(l=1, \ldots, m),
$$


in analogy to the expressions introduced in the proof of Theorem 3.1. Substitution of this expression for the collocation error in the above error equation yields (after division by $h^{p}$ )

$$
\begin{aligned}
\sum_{l=1}^{m} \beta_{n, l} c_{j}^{l-1}= & h \sum_{l=1}^{m} \beta_{n, l} \int_{0}^{c_{j}} K_{1}\left(t_{n, j}, t_{n}+v h\right) v^{l-1} d v \\
& +h \sum_{i=0}^{n-1} \sum_{l=1}^{m} \beta_{i, l} \int_{0}^{1} K_{1}\left(t_{n, j}, t_{i}+v h\right) v^{l-1} d v \\
& +h^{m-p} q_{n, j}-h^{-p} E_{0}\left(t_{n, j}\right) \quad(j=1, \ldots, m) .
\end{aligned}
$$

Proceeding as in the proof of Theorem 3.1, we readily derive, in analogy to (3.7), the discrete Gronwall inequality

$$
\left\|\beta_{n}\right\|_{1} \leq h C_{0} \sum_{i=0}^{n-1}\left\|\beta_{i}\right\|_{1}+\hat{C}_{1} \quad(0 \leq n<r),
$$

where $\hat{C}_{1}:=h^{m-p} C_{1}+h^{q-p} Q_{1}$. This implies that $\left\|\beta_{n}\right\|_{1} \leq \hat{C}_{1} \cdot \exp \left(C_{0} \tau\right), \quad 0 \leq$ $n<r$. Hence, $\left\|\beta_{n}\right\|_{1}$ will remain uniformly bounded as $h \rightarrow 0(r h=\tau)$ if, and only if, $p \leq \min \{m, q\}$.

The case $r \leq n \leq N-1$ is treated in a similar manner; we omit these details, as well as the ones relating to the derivation of the estimate

$$
\left\|e_{i t}\right\|_{\infty} \leq C h^{p}, \quad p=\min \{m, q\} .
$$

Remark. If we employ the (interpolatory) quadrature formulas given in (2.18) to approximate the delay integrals $-\Phi\left(t_{n, j}\right)$, we have $q \geq m$, and hence $p=m$ in (3.11). In other words, in this case the order of convergence of $u$ and $u_{i t}$ is given by (3.2) and (3.3) in Theorem 3.1.

As we mentioned before, the computational form of the collocation method is given by $(2.14)-(2.16)$, with $(2.18)$ for the approximation of the delay integral $-\Phi\left(t_{n, j}\right) \quad(n<r)$, and with

$$
\hat{u}\left(t_{n}+v h\right)=\sum_{k=1}^{m} L_{k}(v) \hat{U}_{n, k}, \quad t_{n}+v h \in I_{n} .
$$

The global convergence property of this continuous implicit $m$-stage DVRK method is described in the following theorem.

Theorem 3.3. Let the assumptions of Theorem 3.2 hold, and assume that the approximations $\hat{\Phi}(t)$ at $t=t_{n, j}(0 \leq n<r)$ are given by the interpolatory quadrature formulas (2.18). Then the error $\hat{e}:=y-\hat{u}$ associated with the collocation solution $\hat{u} \in S_{m-1}^{(-1)}$ (defined by (2.14)-(2.16), (2.18), and (3.12)) satisfies, for all sufficiently small $h=\tau / r$,

$$
\|\hat{e}\|_{\infty} \leq C h^{m} \text {. }
$$

Proof. Since $\hat{e}=y-\hat{u}=(y-u)+(u-\hat{u})$, we have to show that $\|u-\hat{u}\|_{\infty}=$ $O\left(h^{m}\right)$. Consider the integrals occurring in the (exact) collocation equation 
(2.12) (for the linear case (3.1)): using $m$-point interpolatory quadrature formulas based on the collocation points, we have

$$
\int_{0}^{1} K_{1}\left(t_{n, j}, t_{i}+v h\right) u\left(t_{i}+v h\right) d v=\sum_{l=1}^{m} w_{l} K_{1}\left(t_{n, j}, t_{i, l}\right) U_{i, l}+E_{i}^{n, j},
$$

if $0 \leq i<n$, and

$$
\begin{aligned}
\int_{0}^{c_{j}} & K_{1}\left(t_{n, j}, t_{n}+v h\right) u\left(t_{n}+v h\right) d v \\
& =c_{j} \sum_{l=1}^{m} w_{l} K_{1}\left(t_{n, j}, t_{n}+c_{j} c_{l} h\right) u\left(t_{n}+c_{j} c_{l} h\right)+E_{n}^{n, j},
\end{aligned}
$$

where

$$
u\left(t_{n}+c_{j} c_{l} h\right)=\sum_{k=1}^{m} L_{k}\left(c_{j} c_{l}\right) U_{n, k} .
$$

Since the quadrature formulas are of interpolatory type, the corresponding quadrature errors $E_{i}^{n, j}(0 \leq i \leq n)$ satisfy

$$
\left|E_{i}^{n, j}\right| \leq Q h^{m} .
$$

Subtracting (2.14) from (2.12) and using the above quadrature processes, we find, setting $\varepsilon_{n, j}:=U_{n, j}-\hat{U}_{n, j}$,

$$
\begin{aligned}
\varepsilon_{n, j}= & h c_{j} \sum_{l=1}^{m} w_{l} K_{1}\left(t_{n, j}, t_{n}+c_{j} c_{l} h\right) \sum_{k=1}^{m} L_{k}\left(c_{j} c_{l}\right) \varepsilon_{n, k} \\
& +h \sum_{i=0}^{n-1} \sum_{l=1}^{m} w_{l} K_{1}\left(t_{n, j}, t_{i, l}\right) \varepsilon_{i, l} \\
& +Q\left(t_{n, j}\right)+h \sum_{i=0}^{n} E_{i}^{n, j}+h \sum_{i=0}^{n-r} E_{i}^{n-r, j} \quad(j=1, \ldots, m),
\end{aligned}
$$

with

$$
\begin{aligned}
Q\left(t_{n, j}\right):= & h c_{j} \sum_{l=1}^{m} w_{l} K_{2}\left(t_{n, j}, t_{n-r}+c_{j} c_{l} h\right) \sum_{k=1}^{m} L_{k}\left(c_{j} c_{l}\right) \varepsilon_{n-r, k} \\
& +h \sum_{i=0}^{n-r-1} \sum_{l=1}^{m} w_{l} K_{2}\left(t_{n, j}, t_{i, l}\right) \varepsilon_{i, l} .
\end{aligned}
$$

If we define $\varepsilon_{n} \in \mathbf{R}^{m}$ by $\varepsilon_{n}:=\left(\varepsilon_{n, 1}, \ldots, \varepsilon_{n, m}\right)^{T}$, it is then straightforward to show that the norms $\left\|\varepsilon_{n}\right\|_{1}$ satisfy a discrete Gronwall inequality of the form

$$
\left\|\varepsilon_{n}\right\|_{1} \leq h D_{0} \sum_{i=0}^{n-1}\left\|\varepsilon_{i}\right\|_{1}+h D_{1} \sum_{i=0}^{n-r}\left\|\varepsilon_{i}\right\|_{1}+D_{2},
$$


where $D_{2}=O\left(h^{m}\right)$, because of (3.13). Hence, $\left\|\varepsilon_{n}\right\|_{1}=O\left(h^{m}\right)$, and so

$$
\left|u\left(t_{n}+v h\right)-\hat{u}\left(t_{n}+v h\right)\right| \leq \sum_{k=1}^{m}\left|L_{k}(v)\right|\left|\varepsilon_{n, k}\right| \leq \Lambda\left\|\varepsilon_{n}\right\|_{1}=: C h^{m},
$$

where $t_{n}+v h \in I_{n}(n=0, \ldots, N-1)$. Here, $\Lambda$ denotes the Lebesgue constant associated with the points $\left\{c_{j}\right\}$.

We conclude this section with a comment regarding the extension of the results in Theorems 3.1-3.3 to the nonlinear delay integral equation (1.1). Under the assumption of the existence of a (unique) solution $y(t)$ on $I$, the nonlinear analogue of the error equation (3.5) is

$$
\begin{aligned}
e\left(t_{n, j}\right)= & \int_{0}^{t_{n, j}}\left\{k_{1}\left(t_{n, j}, s, y(s)\right)-k_{1}\left(t_{n, j}, s, u(s)\right)\right\} d s \\
& +\int_{0}^{t_{n-r, j}}\left\{k_{2}\left(t_{n, j}, s, y(s)\right)-k_{2}\left(t_{n, j}, s, u(s)\right)\right\} d s
\end{aligned}
$$

$(j=1, \ldots, m)$. If the partial derivatives $\partial k_{i}(t, s, y) / \partial y(i=1,2)$ are continous and bounded on $S \times D$ and $S_{\tau} \times D_{\tau}$, respectively (with $D:=\{y \in \mathbf{R}$ : $|y-y(s)|<Y, s \in I\}$ and $D_{\tau}:=\{y \in \mathbf{R}:|y-y(s)|<Y, s \in[-\tau, T-\tau]\}$, for some $Y<\infty$ ), and if $h>0$ is sufficiently small (assuring the existence of a unique collocation solution $u$ ), then (3.14) may again be written in the form (3.5); the roles of the $K_{i}$ are now assumed by

$$
k_{i}^{(1)}(t, s):=\frac{\partial k_{i}}{\partial y}\left(t, s, z_{i}(s)\right) \quad(i=1,2),
$$

with $z_{i}(s):=\theta_{i} y(s)+\left(1-\theta_{i}\right) u(s), 0 \leq \theta_{i}=\theta_{i}(s) \leq 1$. Hence, the above proofs are readily adapted to deal with the nonlinear case (1.1), and so the convergence results of Theorems 3.1-3.3 remain valid for nonlinear delay integral equations.

\section{LOCAL SUPERCONVERGENCE ON $\Pi_{N}$}

It was shown in $[4,18]$ that if the initial value problem for a delay differential equation,

$$
y^{\prime}(t)=f(t, y(t), y(t-\tau)), \quad t \in I(\tau>0),
$$

is solved by collocation in $S_{m}^{(0)}\left(\Pi_{N}\right)$, with constrained mesh $\Pi_{N}$, and if the collocation points are given by the Gauss (-Legendre) points (i.e., if the $\left\{c_{j}\right\}$ are the zeros of the Legendre polynomial $P_{m}(2 s-1)$ ), then

$$
\max _{1 \leq n \leq N}\left|y\left(t_{n}\right)-u\left(t_{n}\right)\right| \leq C h^{2 m},
$$

provided the exact solution $y$ has continuous derivatives of order $2 m$ on each subinterval $\left(t_{n}, t_{n+1}\right)$. In other words, the Gauss collocation solution for constrained meshes exhibits local superconvergence of order $p^{*}=2 \mathrm{~m}$ at the mesh points (while the global convergence order is $p=m$ ). (The result remains valid for nonconstant delays.)

This superconvergence result does not carry over to the Gauss collocation solution $u \in S_{m-1}^{(-1)}\left(\Pi_{N}\right)$ for the delay Volterra integral equation (1.1): instead 
of (4.1) we only have

$$
\max _{1 \leq n \leq N}\left|y\left(t_{n}\right)-u\left(t_{n}\right)\right|=O\left(h^{m}\right) .
$$

The best possible local superconvergence result is $p^{*}=2 m-1$. However, for the corresponding iterated collocation solution $u_{i t}$, the result (4.1) is again true. The following theorem makes this more precise. Without loss of generality we will assume $T=t_{N}=M \tau$ for some $M \in \mathbf{N}$.

Theorem 4.1. Assume that the given functions in (3.1) and (1.2) are smooth: $g \in C^{m+d}(I), K_{1} \in C^{m+d}(S), K_{2} \in C^{m+d}\left(S_{\tau}\right)$, and $\phi \in C^{m+d}[-\tau, 0]$, for some (given) integer $d$ with $0 \leq d \leq m$. Suppose that the delay integral $\Phi(t)$ (cf. (2.7)) can be evaluated analytically.

If $h=\tau / r$ is sufficiently small, if the collocation parameters $\left\{c_{j}\right\}$ have the orthogonality property

$$
J_{k}:=\int_{0}^{1} s^{k} \prod_{j=1}^{m}\left(s-c_{j}\right) d s=0, \quad k=0, \ldots, d-1 ; J_{d} \neq 0
$$

and if $c_{m}=1$, then the collocation solution $u \in S_{m-1}^{(-1)}\left(\Pi_{N}\right)$ defined by (2.11), (2.12), (2.17) is locally superconvergent at the mesh points whenever $d>0$ :

$$
\max _{1 \leq n \leq N}\left|y\left(t_{n}\right)-u\left(t_{n}\right)\right| \leq C^{*} h^{m+d} .
$$

If $d=m$ (i.e., if the $\left\{c_{j}\right\}$ are the Gauss points, for which $\left.c_{m}<1\right)$, then

$$
\max _{1 \leq n \leq N}\left|y\left(t_{n}\right)-u\left(t_{n}\right)\right| \leq C h^{m}
$$

but

$$
\max _{1 \leq n \leq N}\left|y\left(t_{n}\right)-u_{i t}\left(t_{n}\right)\right| \leq C^{*} h^{2 m}
$$

Here, the iterated collocation solution $u_{i t}$ is determined by (2.13).

Remark. For "classical" Volterra integral equations (i.e., for (1.1) with $k_{2}=0$ ), local superconvergence results for the cases $d=m, d=m-1$ (Radau II points), and $d=m-2$ (Lobatto points) were derived in [8,6, 10]. In [6] these local superconvergence properties were used as the basis for error and stepsize control in a code for such nonlinear integral equations. As will be seen below, the techniques for deriving local superconvergence results for the delay Volterra integral equations (3.1) and (1.1) in many ways closely resemble those employed in the nondelay case $[8,10,9]$; the crucial new element is a generalization of the standard resolvent representation to an analogous one for the solution of (4.7) which takes into account the delay term $F(t)$ (see Lemma 4.3).

Proof of Theorem 4.1. The collocation equation (2.4) (applied to the linear delay equation (3.1)) may be written in the "continuous" form

$$
u(t)=-\delta(t)+g(t)+\int_{0}^{t} K_{1}(t, s) u(s) d s+\int_{0}^{t-\tau} K_{2}(t, s) u(s) d s, \quad t \in I
$$


where the defect $\delta$ vanishes on $X_{N}$ :

$$
\delta\left(t_{n, j}\right)=0, \quad j=1, \ldots, m ; n=0, \ldots, N-1 ;
$$

we also have $\delta(t)=0$ for $t<0$. Moreover, for $0 \leq \nu \leq m+d$, we have that $\delta^{(\nu)}(t)$ is piecewise continuous, with finite jumps at $t=\mu \tau(\mu=0, \ldots$, $M-1)$. The collocation error $e:=y-u$ solves the integral equation

$$
e(t)=\delta(t)+\int_{0}^{t} K_{1}(t, s) e(s) d s+F(t), \quad t \in I,
$$

where

$$
F(t):=\int_{0}^{t-\tau} K_{2}(t, s) e(s) d s \quad \text { if } t \in[\tau, T] .
$$

For $t \in[0, \tau]$, we have (by our assumption on $\Phi(t)) F(t)=0$. The error for the iterated collocation solution $u_{i t}$ is related to $e$ by

$$
e_{i t}(i)=e(t)-\delta(t), \quad t \in I .
$$

Lemma 4.2. Let $i \in[0, \tau]\left(=\left[0, t_{r}\right]\right)$. If

$$
\Phi(t)=\int_{t-\tau}^{0} K_{2}(t, s) \phi(s) d s
$$

is known exactly, then

$$
e(t)=\delta(t)+\int_{0}^{t} R_{1}(t, s) \delta(s) d s .
$$

Here, $R_{1}$ denotes the resolvent kernel associated with the given kernel $K_{1}$ :

$$
R_{1}(t, s)=K_{1}(t, s)+\int_{s}^{t} K_{1}(t, v) R_{1}(v, s) d v, \quad(t, s) \in S .
$$

Proof. For $t \in[0, \tau]$ we have $F(t)=0$, and so the error equation (4.7) reduces to a classical Volterra equation whose (unique) solution is given by (4.10) (see, e.g., [10, pp. 11-13]).

The next lemma contains the central element for the proof of the local superconvergence results of Theorem 4.1; the result may be viewed as a generalization of the resolvent representation of the collocation error (in terms of the defect function $\delta(t))$ for the nondelay case (see, e.g., [9]). Recall that $T=M \tau$ for some positive integer $M$, and set $\xi_{\mu}:=\mu \tau(\mu=0, \ldots, M)$.

Lemma 4.3. Let $\Phi(t)$ satisfy the condition imposed in Lemma 4.2. Then for $t \in\left[\xi_{\mu}, \xi_{\mu+1}\right](1 \leq \mu \leq M-1)$ the collocation error $e(t)$ governed by (4.7) can be expressed in the form

$$
e(t)=\delta(t)+\sum_{i=0}^{\mu} \int_{0}^{t-i \tau} Q_{\mu, i}(t, s) \delta(s) d s,
$$

where the functions $Q_{\mu, i}(t, s)$ depend on the given kernel functions $K_{i}(t, s)$ $(i=1,2)$, and their degree of smoothness is determined by that of $K_{1}$ and $K_{2}$. 
Proof. Consider first the interval $[\tau, 2 \tau]\left(=\left[\xi_{1}, \xi_{2}\right]: \mu=1\right)$. Using the result (4.10) of Lemma 4.2 (with $\delta(s)$ replaced by $\delta(s)+F(s)$ ), we can write the error equation (4.7) as

$$
e(t)=\delta(t)+\int_{0}^{t} R_{1}(t, s) \delta(s) d s+F(t)+\int_{0}^{t} R_{1}(t, s) F(s) d s,
$$

with $F(t)$ given by (4.8). Since $t-\tau$ is in $[0, \tau]$, we find

$$
\begin{aligned}
F(t) & =\int_{0}^{t-\tau} K_{2}(t, s) \cdot\left\{\delta(s)+\int_{0}^{s} R_{1}(s, v) \delta(v) d v\right\} d s \\
& =\int_{0}^{t-\tau} K_{2}(t, s) \delta(s) d s+\int_{0}^{t-\tau}\left(\int_{v}^{t-\tau} K_{2}(t, s) R_{1}(s, v) d s\right) \delta(v) d v .
\end{aligned}
$$

Set

$$
F(t):=\int_{0}^{t-\tau} G_{1,1}(t, s) \delta(s) d s
$$

where

$$
G_{1,1}(t, s):=K_{2}(t, s)+\int_{s}^{t-\tau} K_{2}(t, v) R_{1}(v, s) d v .
$$

(Note that the resolvent kernel $R_{1}(t, s)$ inherits the smoothness properties of $K_{1}(t, s)$.) Therefore, since $F(t)=0$ on $[0, \tau]$,

$$
\begin{aligned}
\int_{0}^{t} R_{1}(t, s) F(s) d s & =\int_{\tau}^{t} R_{1}(t, s)\left(\int_{0}^{s-\tau} G_{1,1}(s, v) \delta(v) d v\right) d s \\
& =\int_{0}^{t-\tau}\left(\int_{v+\tau}^{t} R_{1}(t, s) G_{1,1}(s, v) d s\right) \delta(v) d v \\
& =: \int_{0}^{t-\tau} G_{1,2}(t, s) \delta(s) d s .
\end{aligned}
$$

Hence, (4.11) follows with

$$
Q_{1,1}(t, s):=G_{1,1}(t, s)+G_{1,2}(t, s) .
$$

For $t \in[\mu \tau,(\mu+1) \tau](2 \leq \mu \leq M-1)$, the result (4.11) is established in a similar manner by a simple induction step, using the equations (4.7), (4.8), and (4.11) in $[(\mu-1) \tau, \mu \tau]$. Since $\delta(v)=0$ for $v<0$, we have the identity (using Dirichlet's formula)

$$
\begin{aligned}
\int_{0}^{t-\tau} K_{2}(t, & s)\left(\int_{0}^{s-i \tau} Q_{\mu, i}(s, v) \delta(v) d v\right) d s \\
= & \int_{i \tau}^{t-\tau} K_{2}(t, s)\left(\int_{0}^{s-i \tau} Q_{\mu, i}(s, v) \delta(v) d v\right) d s \\
= & \int_{0}^{t-(i+1) \tau}\left(\int_{v+i \tau}^{t-\tau} K_{2}(t, s) Q_{\mu, i}(s, v) d s\right) \delta(v) d v .
\end{aligned}
$$

Details are left to the reader. 
The results of Lemma 4.2 and Lemma 4.3 and the companion expression for the iterated collocation error (which follows from (4.9)),

$$
e_{i t}(t)=\sum_{i=0}^{\mu} \int_{0}^{t-i \tau} Q_{\mu, i}(t, s) \delta(s) d s, \quad t \in\left[\xi_{\mu}, \xi_{\mu+1}\right],
$$

form the basis for the local superconvergence results in Theorem 4.1. Let $t=$ $t_{n} \in\left[\xi_{\mu}+h, \xi_{\mu+1}\right]$. Observing that $t_{n}-i \tau=t_{n}-i r h=(n-i r) h$, we obtain

$$
\begin{aligned}
e\left(t_{n}\right) & =\delta\left(t_{n}\right)+\sum_{i=0}^{\mu} \int_{0}^{t_{n}-i \tau} Q_{\mu, i}\left(t_{n}, s\right) \delta(s) d s \\
& =\delta\left(t_{n}\right)+h \sum_{i=0}^{\mu} \sum_{k=0}^{n-i r-1} \int_{0}^{1} \Psi_{\mu, i}^{[n]}\left(t_{k}+v h\right) d v,
\end{aligned}
$$

with

$$
\Psi_{\mu, i}^{[n]}\left(t_{k}+v h\right):=Q_{\mu, i}\left(t_{n}, t_{k}+v h\right) \delta\left(t_{k}+v h\right) .
$$

If we now replace each of the integrals in the above expression by the sum of its interpolatory $m$-point quadrature formula (with the abscissas coinciding with the collocation points $\left.t_{k}+c_{j} h, j=1, \ldots, m\right)$ and the corresponding quadrature error $E_{\mu, i}^{[n]}$, we obtain, since $\delta(t)=0$ for $t \in X_{N}$ (cf. (4.6)) implies $\Psi_{\mu, i}^{[n]}\left(t_{k}+c_{j} h\right)=0$,

$$
e\left(t_{n}\right)=\delta\left(t_{n}\right)+h \sum_{i=0}^{\mu} \sum_{k=0}^{n-i r-1} E_{\mu, i}^{[n]} \quad(0 \leq \mu<n \leq \mu+1 \leq M),
$$

where $M \tau=T$. The orthogonality condition (4.2) implies that these (interpolatory) $m$-point quadrature formulas all possess the degree of precision $m+d$. Thus, since the integrands $\Psi_{\mu, i}^{[n]}\left(t_{k}+v h\right)$ are smooth for $v \in[0,1]$, the quadrature errors in (4.13) can be bounded by $\left|E_{\mu, i}^{[n]}\right| \leq \tilde{C} h^{m+d}$ with some finite constant $\tilde{C}$ not depending on $h$. Finally, noting that $M \tau=M \cdot r h=T=N h$ and that therefore the number of terms in the above sums is $\mathscr{O}(N)$, we arrive at the estimates $\left|e\left(t_{n}\right)\right| \leq\left|\delta\left(t_{n}\right)\right|+c h^{m+d}$, and, by (4.12), $\left|e_{i t}\left(t_{n}\right)\right| \leq C h^{m+d}$ $(n=1, \ldots, N)$.

If $c_{m}=1$, then $t_{n-1}+c_{m} h=t_{n} \in X_{N}$, and thus $\delta\left(t_{n}\right)=0,1 \leq n \leq N$. This proves (4.3). Note that under this constraint on $c_{m}$ we have $d \leq m-1$, with $d=m-1$ if, and only if, the $\left\{c_{j}\right\}$ are the Radau II points, i.e., the zeros of $P_{m}(2 s-1)-P_{m-1}(2 s-1)$. For continuous $u$ (corresponding to the choice $\left.c_{1}=0, c_{m}=1 ; m \geq 2\right)$, the optimal value of $d$ in (4.3) is $d=m-2$.

The maximum value of $d$ in the orthogonality condition (4.2), $d=m$, occurs if, and only if, the collocation parameters are the Gauss (-Legendre) points in $(0,1)$. For these points we have $0<c_{1}<\cdots<c_{m}<1$, and hence $\delta\left(t_{n}\right) \neq 0$. It is easily seen from the definition of the defect $\delta$ that, in general, we can do no better than $\delta(t)=O\left(h^{m}\right)$ for $t \notin X_{N}$. (Consider (3.1) with $K_{i}=$ const,$\phi=$ const ; in this case $\delta \in S_{m}^{(0)}\left(\Pi_{N}\right)$, i.e., is piecewise continuous of degree $m$, vanishing on $X_{N} \not \supset \Pi_{N}$.) This yields (4.4). The estimate (4.5) for the iterated collocation solution follows from (4.9) (or (4.12)). 
The proofs of Theorems 3.2, 3.3, and 4.1 readily suggest that the local superconvergence results of Theorem 4.1 are also true for the discretized collocation solution $\hat{u} \in S_{m-1}^{(-1)}\left(\Pi_{N}\right)$ defined by (2.14)-(2.16), (2.18), and (3.12), and for the corresponding iterated collocation solution $\hat{u}_{i t}$ (cf. (2.20)), provided the quadrature approximations to the delay integrals

$$
\begin{aligned}
\Phi\left(t_{n}\right) & =-\int_{t_{n-r}}^{0} k_{2}\left(t_{n}, s, \phi(s)\right) d s \\
& =-h \sum_{i=n-r}^{-1} \int_{0}^{1} k_{2}\left(t_{n}, t_{i}+v h, \phi\left(t_{i}+v h\right)\right) d v
\end{aligned}
$$

are given by

$$
\hat{\boldsymbol{\Phi}}\left(t_{n}\right)=-h \sum_{i=n-r}^{-1} \sum_{l=1}^{m} w_{l} k_{2}\left(t_{n}, t_{i, l}, \phi\left(t_{i, l}\right)\right) \quad(0 \leq n<r) .
$$

We leave the details of the proof to the reader, but summarize these results in

Theorem 4.4. Let the assumptions of Theorem 4.1 hold, and assume that the approximations to the delay integrals $\Phi\left(t_{n, j}\right)$ and $\Phi\left(t_{n}\right)$ (where $0 \leq n<r$ ) are given by the quadrature processes (2.18) and (4.14), respectively.

If $h=\tau / r$ is sufficiently small and if the orthogonality condition (4.2) holds, then the solution $\hat{u}$ given by the continuous implicit DVRK method \{(2.14)(2.16), (3.12)\} has the property

$$
\max _{1 \leq n \leq N}\left|y\left(t_{n}\right)-\hat{u}\left(t_{n}\right)\right| \leq C^{*} h^{m+d},
$$

provided that $c_{m}=1$ (and thus $d \leq m-1$ ).

If the $\left\{c_{j}\right\}$ are the Gauss points in $(0,1)$, then

$$
\max _{1 \leq n \leq N}\left|y\left(t_{n}\right)-\hat{u}\left(t_{n}\right)\right| \leq C h^{m},
$$

while

$$
\max _{1 \leq n \leq N}\left|y\left(t_{n}\right)-\hat{u}_{i t}\left(t_{n}\right)\right| \leq C^{*} h^{2 m}
$$

Finally, we remark that Theorems 4.1 and 4.4 can be extended to the nonlinear Volterra equation (1.1), as follows. Instead of (4.7), the equation for the collocation error $e$ now has the form

$$
e(t)=\delta(t)+\int_{0}^{t}\left(k_{1}(t, s, y(s))-k_{1}(t, s, u(s))\right) d s+F(t)
$$

where

$$
F(t):=\int_{0}^{t-\tau}\left(k_{2}(t, s, y(s))-k_{2}(t, s, u(s))\right) d s .
$$


Under appropriate differentiability and boundedness conditions for $k_{1}$ and $k_{2}$ we then find, setting $u(s)=y(s)-e(s)$,

$$
\begin{aligned}
k_{i}(t, s, y(s))-k_{i}(t, s, u(s))= & \frac{\partial k_{i}}{\partial y}(t, s, y(s)) \cdot e(s) \\
& +\frac{1}{2} \frac{\partial^{2} k_{i}}{\partial y^{2}}\left(t, s, z_{i}(s)\right) \cdot e^{2}(s),
\end{aligned}
$$

where $z_{i}$ is between $y$ and $u$. Since the global convergence of $u$ (and $\hat{u}$ ) has already been established, we know that

$$
\left\|e^{2}\right\|_{\infty}=O\left(h^{2 m}\right) \text { for any }\left\{c_{j}\right\} \text {. }
$$

The remaining part of the proofs (both for $u$ and $\hat{u}$ ) once more makes use of the techniques described before.

\section{CONCLUding REMARKS}

It should be possible to combine the techniques used in $[4,5,18]$ for delay differential equations with the ones employed in this paper in order to extend the above results to Volterra integral equations with variable delays (e.g., to (1.4)) or with multiple delays ([7]; see also [16] for delay differential equations with multiple (constant) delays). Volterra integral equations with state-dependent delays appear to be much more complex to deal with (compare [12]).

In [19] Vermiglio considered, among other things, the P-stability of collocation methods for Volterra integral equations with delay. In particular, it was shown that if the collocation parameters $\left\{c_{j}\right\}$ are such that they yield an $A$-stable collocation method for an ordinary differential equation, then the correponding (discretized) collocation method for the delay integral equation (1.1) is $P$-stable. As we have seen, for delay integral equations collocation at the Gauss points does not lead to local superconvergence at $t=t_{n}$; it is the corresponding iterated collocation solution that exhibits $O\left(h^{2 m}\right)$-convergence at the mesh points. Is the iterated collocation solution $\hat{u}_{i t}$ corresponding to the Gauss collocation solution $\hat{u}$ also $P$-stable? This question will be studied in a sequel to the present paper.

\section{BIBLIOGRAPHY}

1. H. Arndt and C. T. H. Baker, Runge-Kutta formulae applied to Volterra functional equations with fixed delay, Numerical Treatment of Differential Equations: 4th Internat. Seminar NUMDIFF (Halle-Wittenberg, 1987), Teubner-Texte Math., vol. 104, Leipzig, 1988, pp. 19-30.

2. C. T. H. Baker and M. S. Derakhshan, $R$-K formulae applied to Volterra equations with delay, J. Comput. Appl. Math. 29 (1990), 293-310.

3. J. Banaś, An existence theorem for nonlinear Volterra integral equation with deviating argument, Rend. Circ. Mat. Palermo (2) 35 (1986), 82-89.

4. A. Bellen, One-step collocation for delay differential equations, J. Comput. Appl. Math. 10 (1984), 275-283.

5. _ Constrained mesh methods for functional differential equations, Delay Equations, Approximation and Applications (G. Meinardus and G. Nürnberger, eds.), Internat. Ser. Numer. Math., vol. 74, Birkhäuser Verlag, Basel, 1985, pp. 52-70. 
6. J. G. Blom and H. Brunner, The numerical solution of nonlinear Volterra integral equations of the second kind by collocation and iterated collocation methods, SIAM J. Sci. Statist. Comput. 8 (1987), 806-830.

7. J. M. Bownds, J. M. Cushing, and R. Schutte, Existence, uniqueness, and extendibility of solutions of Volterra integral systems with multiple, variable lags, Funkcial. Ekvac. 19 (1976), 101-111.

8. H. Brunner, Iterated collocation methods and their discretizations for Volterra integral equations, SIAM J. Numer. Anal. 21 (1984), 1132-1145.

9. __ On discrete superconvergence properties of spline collocation methods for nonlinear Volterra integral equations, J. Comput. Math. 10 (1992), 348-357.

10. H. Brunner and P. J. van der Houwen, The numerical solution of Volterra equations, CWI Monograph 3, North-Holland, Amsterdam, 1986.

11. B. Cahlon, On the numerical stability of Volterra integral equations with delay argument, J. Comput. Appl. Math. 33 (1990), 97-104.

12. B. Cahlon and L. J. Nachman, Numerical solutions of Volterra integral equations with a solution dependent delay, J. Math. Anal. Appl. 112 (1985), 541-562.

13. K. L. Cooke, An epidemic equation with immigration, Math. Biosci. 29 (1976), 135-158.

14. R. Esser, Numerische Behandlung einer Volterraschen Integralgleichung, Computing 19 (1978), 269-284.

15. S. Sugiyama, On functional integral equations, Mem. School Sci. Engrg. Waseda Univ. 41 (1977), 135-153.

16. L. Torelli and R. Vermiglio, On the stability of continuous quadrature rules for differential equations with $r$ delays, IMA J. Numer. Anal. 13 (1993), 291-302.

17. P. Vâță, Convergence theorems of some numerical approximation schemes for the class of non-linear integral equations, Bul. Univ. Galaţi Fasc. II Mat. Fiz. Mec. Teoret. 1 (1978), 25-33.

18. R. Vermiglio, A one-step subregion method for delay differential equations, Calcolo 22 (1985), 429-455.

19. 61 (1992), 561-577.

20. M. Zennaro, Natural extensions of Runge-Kutta methods, Math. Comp. 46 (1986), 119-133.

Department of Mathematics and Statistics, Memorial University of Newfoundland,

St. John's, Newfoundland, Canada A1C 5S7

E-mail address: hbrunner@kean.ucs.mun.ca 